\title{
Effectiveness of Intravenous Immunoglobulin Plus Plasmapheresis on Antibody-mediated Rejection or Thrombotic Microangiopathy in Iranian Kidney Transplant Recipient
}

\author{
Simin Dashti-Khavidaki ${ }^{1, *}$; Lida Shojaie ${ }^{2}$; Amin Hosni ${ }^{2}$; Mohammad Reza Khatami ${ }^{1}$; Atefeh \\ Jafari $^{2}$ \\ ${ }^{1}$ Nephrology Research Center, Tehran University of Medical Sciences, Tehran, Iran \\ ${ }^{2}$ Department of Clinical Pharmacy, Tehran University of Medical Sciences, Tehran, Iran \\ ${ }^{*}$ Corresponding author: Simin Dashti-Khavidaki, Nephrology Research Center, Tehran University of Medical Sciences, Tehran, Iran. Tel: +98-2166581568, Fax: +98-2166581568, \\ E-mail:dashtis@sina.tums.ac.ir
}

Received: January 17, 2015; Accepted: February 9, 2015

\begin{abstract}
Background: Antibody mediated rejection (AMR) and thrombotic microangiopathy (TMA) after kidney transplantation are difficult to differentiate most of the times and both play important roles in kidney allograft loss. Common treatment strategies of these two conditions include plasmapheresis, intravenous immunoglobulin (IVIG) and rituximab.

Objectives: This study was designed to assess the efficacy of routine treatment of AMR/TMA in Iranian kidney transplant recipients, which comprises of plasmapheresis and IVIG.

Patients and Methods: This one-year cross-sectional study was performed in the Kidney Transplantation Ward of Imam-Khomeini Hospital Complex, Tehran, Iran. All kidney transplant recipients who were administered plasmapheresis and IVIG to treat definite or suggested AMR or TMA were assessed clinically and also evaluated on laboratory data.

Results: During 2014, we encountered five patients with suspicious AMR or TMA at our kidney transplant center. Renal biopsy was performed for two of them, suggesting AMR for one patient and TMA for another patient. All patients were treated with plasmapheresis plus IVIG. In this center, as a routine practice, the cumulative dose of $2 \mathrm{~g} / \mathrm{kg}$ of IVIG was divided to $300-400 \mathrm{mg} / \mathrm{kg}$ after each plasmapheresis. Only one out of the five patients showed response, albeit not completely.

Conclusions: Due to daily plasmapheresis within the first several days after AMR or TMA, administering high amounts of the cumulative dose of IVIG after plasmapheresis may result in high amounts of IVIG withdrawal by plasmapheresis and response failure. Our suggestion is to reduce the IVIG dose after each plasmapheresis to $100 \mathrm{mg} / \mathrm{kg}$ (i.e. replacement dose) to reach a cumulative dose of $2 \mathrm{~g} /$ $\mathrm{Kg}$. If plasmapheresis treatment is initiated sooner than the completion of the IVIG cumulative dose of $2 \mathrm{~g} / \mathrm{kg}$, the remaining dose can be administered during one injection.
\end{abstract}

Keywords:Graft Rejection; Immunoglobulins, Intravenous; Kidney Transplantation; Plasmapheresis; Thrombotic Microangiopathy

\section{Background}

Kidney transplantation is the treatment of choice for patients who suffer end-stage renal disease (ESRD). Survival and quality of life in renal transplant recipients are more than in dialysis patients $(1,2)$. Unfortunately, organ availability for kidney transplantation is restricted by the growing number of patients waiting for this treatment (3). Additionally, several complications occur after kidney transplantation, including surgical complications (e.g. fluid collections, urinoma, lymphocele, hematoma, and abscess), incisional hernia, acute kidney injury, infections and allograft rejection (4). Among these complications, the management of acute rejection is very important, because each rejection that is not controlled will destroy the allograft and increase patient's morbidity and mortality (2). There are two international classifications for acute allograft rejection: T cell-mediated acute rejection and antibody-mediated acute rejection (AMR). The presentation of acute allograft rejection has changed significantly in the past decade. T cell-mediated acute rejection was more studied than AMR over the past several years. The use of potent immunosuppressive agents has greatly reduced the rate of acute cellular rejection. Consequently, the 1-year graft survival has significantly improved, following kidney transplantation. In recent years, acute and chronic AMR are playing serious roles in kidney allograft loss and are considered as important causes that limit long-term outcomes of kidney transplantation $(2,5,6)$. Within the few days after kidney transplantation, early acute AMR occurs in transplant recipients with anti-HLA or donor specific antibodies (DSA) (7). Rejection phenotypes and outcomes are more complex in clinical practice than in the theory. Rejection can be diagnosed by renal

Copyright ( ) 2015, Nephrology and Urology Research Center. This is an open-access article distributed under the terms of the Creative Commons Attribution-NonCommercial 4.0 International License (http://creativecommons.org/licenses/by-nc/4.0/) which permits copy and redistribute the material just in noncommercial usages, provided the original work is properly cited 
biopsy findings, such as C4d deposition in the peritubular capillaries and histological features of inflammation, allograft dysfunction, and serologic evidence of circulating antibodies (2). Despite significant advances in recent years, AMR cannot be diagnosed only according to the clinical features. Several differential diagnoses have to be considered when AMR is suspicious, which include acute ischemic injury, acute calcineurin inhibitor (CNI) toxicity, infections and thrombotic microangiopathy (TMA). The TMA is always the most important differential diagnosis of AMR (8). The gold standard for exact diagnosis is renal biopsy (9); however, in many patients, performing kidney biopsy is not feasible due to the patient's low platelet count and lack of personal satisfaction. In these situations, the best treatment must be selected to cover both AMR and TMA.

\section{Objectives}

This study was designed to assess the efficacy of routine treatment of AMR/TMA in Iranian kidney transplant recipients, comprising of plasmapheresis and intravenous immunoglobulin (IVIG).

\section{Patients and Methods}

\subsection{Study Design}

This 1-year cross-sectional study was performed at the Kidney Transplantation Ward of Imam-Khomeini Hospital Complex, affiliated to Tehran University of Medical Sciences, Tehran, Iran from 1st January 2014. All kidney transplant recipients, who were administered plasmapheresis and IVIG to treat definite or suggested AMR or TMA, were assessed.

\subsection{Immunosuppressive/Prophylaxis Protocol}

All patients in this transplant ward receive rabbit antithymocyte globulin (rATG), as induction therapy, with a cumulative dose of at least $6 \mathrm{mg} / \mathrm{kg}$. They are also administered methylprednisolone $500 \mathrm{mg}$ on the transplantation day and 250 and $125 \mathrm{mg}$ on the next two days after transplantation, which is continued with oral prednisolone $1 \mathrm{mg} / \mathrm{kg} /$ day, with rapid taper down during the next days, to reach the dose of $5 \mathrm{mg} /$ day after month one from transplantation. They also receive a CNI (mostly tacrolimus) and mycophenolate mofetil as maintenance immunosuppressive regimen.

All patients receive intravenous ganciclovir/oral valganciclovir, oral co-trimoxazole and clotrimazole for routine viral, pneumocystis pneumonia, and fungal prophylaxis.

\subsection{Data Gathering}

All patients who were suspicious for AMR or TMA were evaluated. Patients' demographic and clinical data (sex, age, weight, height, cause of ESRD), laboratory findings (including serum creatinine and urea concentrations, urine volume, serum electrolytes, complete blood counts and serum lactate dehydrogenase (LDH) concentration), were gathered in designed forms.

\subsection{Ethics}

The study protocol was approved by local Ethics Committee of Tehran University of Medical Sciences, Tehran, Iran. All patients or their representatives provided their informed consent and were assured that their information will be published anonymously.

\section{Results}

Five kidney transplant patients were treated for suspicious/definite AMR or TMA during the year 2014 at this center.

\subsection{Case 1}

The patient S.T., a 56-year-old female, with a history of ESRD with undiagnosed cause, was admitted in February 2014 for a second renal transplantation. She had a history of renal transplant with hyperacute rejection 6 years ago. Her present kidney was from a 32-year-old living male. This patient had at least 10 pregnancies. His panel reaction antibody (PRA) was 10\%. After induction, her maintenance immunosuppressive regimen included tacrolimus, prednisolone and mycophenolate mofetil. Her creatinine was decreased from 5.6 to $0.9 \mathrm{mg} / \mathrm{dl}$, within 5 days after transplantation; however, on day 6 after transplantation, her urine output was significantly decreased and her serum creatinine level rose. Her platelet count dropped from 173000 to 56000 cells $/ \mathrm{mm}^{3}$. Our concern regarded the TMA and possibility of acute rejection, although we were unable to perform allograft biopsy, because of her low platelet count. Two days later, she received IVIG, with a cumulative dose of $2.3 \mathrm{~g} / \mathrm{kg}$ ideal body weight, for 5 days, which is equal to approximately 460 $\mathrm{mg} / \mathrm{kg} /$ day. Plasmapheresis and IVIG were prescribed for seven sessions. Peripheral blood smear was negative for the presence of schistocytes. Concomitant with IVIG therapy, plasmapheresis was performed daily for four sessions and thereafter, every other day, for three sessions. The IVIG doses were infused after plasmapheresis treatment. Due to lack of improvement in kidney function and platelet count, tacrolimus was stopped 16 days after transplantation. Treatment with plasmapheresis and IVIG was not effective in this patient and therefore was discontinued. Due to uncontrolled fever and patient's illness, nephrectomy was performed in her case.

\subsection{Case 2}

The patient A.D., a 15-year-old male, with a history of ESRD of unknown origin, was admitted in May 2014 for preemptive kidney transplantation from a 14-year-old deceased male. His PRA was negative. His maintenance immunosuppressive regimen included tacrolimus, my- 
Dashti-Khavidaki S et al.

cophenolate mofetil and prednisolone. After transplantation, his urine output and serum creatinine concentration did not improve. Although his diethylene triamine pentacaetic acid (DTPA) radionuclide scan suggested acute rejection, renal biopsy could not be performed. Plasmapheresis and high-dose of IVIG ( $2 \mathrm{~g} / \mathrm{kg}$ ) were prescribed 2 days after transplantation. He was treated with plasmapheresis for 9 consecutive days and his IVIG was divided to these 9 days after each plasmapheresis session (i.e. about $222 \mathrm{mg} / \mathrm{kg}$ after each plasmapheresis). He was discharged from the hospital with a serum creatinine level similar to his pretransplant value.

\subsection{Case 3}

The patient A.R., a 40-year-old male, with a history of ESRD with undetermined origin, was admitted in June 2014. He received the kidney from a 33-year-old deceased male. His PRA was negative. His maintenance regimen included tacrolimus, mycophenolate mofetil and prednisolone. During the first few days he showed good allograft functioning. However, 3 days after transplantation, serum LDH level increased and platelet count decreased significantly. His platelet count did not increase despite rATG discontinuation. Renal biopsy was not performed in this patient. Plasmapheresis was started, with assumption of TMA for five daily sessions. Four days after plasmapheresis initiation, LDH decreased and platelet count increased. At day 18 from transplantation, his serum creatinine concentration increased from $1.5 \mathrm{mg} / \mathrm{dl}$ to 1.9 and $2.4 \mathrm{mg} / \mathrm{dl}$, during two days. Therefore, his plasmapheresis was continued additionally for four sessions every other day and $1.5 \mathrm{~g} / \mathrm{kg}$ of IVIG was divided to four doses (375 mg/ $\mathrm{kg}$ each) on the days that patient did not undergo plasmapheresis. After this treatment, his creatinine was decreased to $1.7 \mathrm{mg} / \mathrm{dl}$ and relative improvement occurred.

\subsection{Case 4}

The patient M.R., a 44-year-old male with a history of ESRD with undetermined origin, was admitted in September 2014 and received the kidney from a 37-year-old deceased female. His PRA was negative before transplantation. His maintenance regimen included tacrolimus, mycophenolate mofetil and prednisolone. After transplantation, his serum creatinine was not decreased and he was anuric. His first renal biopsy, which was performed 5 days after transplantation, showed C4d deposition in peritubular capillaries. Plasmapheresis was performed daily for 5 days and every other day, for five additional sessions. The IVIG with a cumulative dose of $2.1 \mathrm{~g} / \mathrm{kg}$ was administered during 8 consecutive days (i.e. about $263 \mathrm{mg} / \mathrm{kg}$ each day), starting the day before plasmapheresis initiation. However, there was no improvement in his renal function. The second biopsy that was done 11 days after transplantation revealed necrosis and vascular congestion, associated with AMR. Because of no improvement in his renal function, the third biopsy was done on day 16 after transplantation and showed more than $90 \%$ glomerular necrosis.

\subsection{Case 5}

The patient H.N., a 57-year-old female with a history of ESRD because of autosomal dominant polycystic kidney disease, was admitted in October 2014. Her donor was a 58-year-old deceased female. Her maintenance regimen included tacrolimus, mycophenolate mofetil and prednisolone. Serum creatinine decreased slowly after kidney transplantation. At day 11 after transplantation, the DTPA scan was requested because of delayed graft function, suggesting acute rejection. The biopsy was performed at day 12 , showing glomerular basement membrane thickening and subendothelial widening that was compatible with TMA. After that, platelet count decreased from 100000 to 39000 cells/microliter and serum LDH level increased. Therefore, she received methylprednisolone pulses for 3 days. She received a cumulative dose of $2.3 \mathrm{~g} /$ $\mathrm{kg}$ IVIG during 4 consecutive days (i.e. about $383 \mathrm{mg} / \mathrm{kg}$ at each doses), started with plasmapheresis treatment. Plasmapheresis was performed for a cumulative 15 sessions, six of which were daily and the others every 2 - 3 days. The patient's general condition was deteriorated and despite nephrectomy and antibiotic therapy, the patient unfortunately expired in a sepsis tableau.

\section{Discussion}

During recent years, the use of effective immunosuppressants resulted in the decrease of the rate of acute cellular rejection. However, AMR has become an important cause of graft loss during the initial weeks to months after transplantation (7). The incidence of acute graft loss in AMR is greater than acute cellular rejection (7). In patients with high levels of DSA, the incidence of graft loss in the first month after transplantation may be as high as $40 \%$, while this rate is less than $10 \%$ in patients with a negative DSA $(7,10)$. Early aggressive treatment of AMR may be required to prevent graft loss (7). Dehydration or elevated blood levels of CNIs can also cause an increase in serum creatinine concentration. Therefore, we would be cautious to differentiate AMR from these situations. Although renal biopsy and measurement of the serum DSA are helpful, they are not always readily practical or available in the clinical settings (7). Performing renal biopsy is sometimes limited due to patients' severe thrombocytopenia. The TMA is the major differential diagnosis for AMR (8). Therefore, it is indicated to choose a treatment regimen that is helpful for both conditions. During year 2014, we encountered five patients with suspicious AMR or TMA at our kidney transplant center. Renal biopsy was performed for two of them, suggesting AMR in one patient and TMA for the other. Therefore, we treated them with plasmapheresis plus IVIG; however, only one out of these five patients showed response albeit not completely. 
Five treatment modalities have been proposed to manage AMR. The first strategy is to control B-cell activities by removal or dilution of antibodies. We can use plasmapheresis or immunoadsorption for removal of antibodies. By administration of IVIG, antibodies against the graft can be diluted. The second option is to inhibit or deplete of B-cells using antibodies, such as rituximab, that act against CD20 in B-cells surface. The third way is to reduce T-cells. Diminished T-cells can decrease B-cell counts. Inhibitors of T-cell division (mycophenolate mofetil and steroids), inhibitors of IL-2 signaling to Tcells (CNIs), and T-cell depleting agents such as rATG are three options that decrease T-cells. A fourth target for the managing of AMR is to diminish the plasma cells that produce antibodies. Proteasome inhibitors, such as bortezomib can be administered for this goal. Antibodies that are produced by plasma cell will become bound to graft and activate complement cascade. Therefore, the fifth target that has recently been introduced is the fixation of complement, which is activated by antibodies. Eculizumabis, a C5 inhibitor, is an option that reduces the dissemination of the complement cascade, even after antibodies have bound to the graft $(1,6,11)$.

Despite these various options for AMR treatment, there is no consensus over which treatment must be selected first in transplanted patients with $\operatorname{AMR}(6-8,11,12)$. The importance of this issue is more apparent when we could not perform biopsy, as a gold standard for detecting AMR. On the other hand, in several situations we could not distinguish between AMR and TMA, according to the clinical features. Therefore, designing a useful treatment strategy that could be used in both AMR and TMA is very crucial. At present, there is insufficient data to guide us for adequate treatment. Most of the studies demonstrated the mechanisms of AMR and related therapies. Nevertheless, the first line of therapy was not indicated by any study $(7,11)$. The systematic review conducted by Roberts et al. demonstrated that the optimal treatment for AMR remains unknown. They recommended a combination treatment for the management of AMR that is associated with multiple pathophysiologic pathways. This combination includes plasmapheresis, IVIG, immunosuppressants, rituximab, bortezumib and eculizumab (13). As seen, this proposed strategy is too expensive to be applied for all patients with definite or possible AMR.

The management of TMA has also been reviewed in many studies. Almost always, plasma exchange is the first line of TMA treatment. The use of corticosteroids is recommended in TMA patients with neurological or cardiovascular involvement. With these therapies, there is still a high mortality rate of $10-20 \%$ and a high relapse rate, of approximately 20 - 50\%. The effectiveness of rituximab in decreasing TMA relapse and hospitalization duration has been confirmed in a series of studies (14-18). A cohort study that has been performed by Westwood et al. revealed that early administration of rituximab for thrombotic thrombocytopenic purpura (TTP) (within 3 days) was associated with faster remission, fewer plasma exchange sessions and shorter duration of hospital stay (14). The efficacy and safety of rituximab in severe TTP was shown by Froissart et al. in adults, who responded poorly to therapeutic plasma exchange. One-year relapses and hospitalization duration were decreased in patients who were treated with rituximab, compared with historical controls, who were treated with plasmapheresis (18).

In the present study, the effectiveness of plasmapheresis and IVIG were investigated in Iranian kidney transplant recipients, with suspicious/definite AMR or TMA. Assessing donor specific antibody is not performed in Iranian laboratories. Kidney biopsy is not performed in patients with thrombocytopenia in most Iranian kidney transplant wards, due to legal considerations and fear of bleeding and its ominous consequences. Due to similar clinical and laboratory features of AMR and TMA, including increased serum creatinine concentration, decreased urine output and platelet count, and increased serum LDH levels, most clinicians consider both situations when selecting treatment. According to the high cost of rituximab and lack of Iranian insurance coverage of rituximab by nephrologist's order, a limited number of patients can afford its cost. Therefore, rituximab cannot be easily used as first option in these situations in kidney transplant patients, which determines Iranian nephrologists to usually start AMR or TMA treatment with plasmapheresis plus IVIG. Applying these therapies in our patients cohort showed that only one out of five patients (20\%), showed only partial response to these treatments. The AMR and TMA were confirmed only in two patients by biopsy (each in one patient). According to the literature, it seems better to start combination treatments for both AMR and TMA, with common regimen of plasmapheresis plus IVIG, plus rituximab. Almost all patients with TMA are at risk of relapse $(19,20)$. In transplanted patients we aim to decrease the relapse rate, because each TMA episode could result in graft failure (20), and rituximab is the required agent (14-18). The time of initiation of IVIG and plasmapheresis was different between our patients. According to the study by Jodele et al. (21) early plasmapheresis could be effective in TTP patients even with multiorgan damage. Therefore, the early use of plasmapheresis and rituximab are recommended in situation in which it is difficult to distinguish between TMA and AMR. However, according to the high cost and lack of insurance coverage of rituximab for kidney transplant recipients, almost none of our patients could benefit from this drug. Another explanation for the high rate of treatment failure in our patients may be the ineffectiveness of IVIG, possibly due to its removal by plasmapheresis. In most studies, it is recommended to administer IVIG with maintenance dose of $100 \mathrm{mg} / \mathrm{kg}$ after each plasmapheresis, and when plasmapheresis was stopped, the remaining dose of IVIG, to a cumulative dose of $2 \mathrm{~g} / \mathrm{kg}$, could be administered once (11). In our center, as a routine practice of nephrology attending that has reached to nephrology 
fellows as well, the cumulative dose of $2 \mathrm{~g} / \mathrm{kg}$ of IVIG is divided into $300-400 \mathrm{mg} / \mathrm{kg}$ after each plasmapheresis. Due to daily plasmapheresis within the first few days after AMR or TMA, this IVIG administration approach may result in high amount of IVIG withdrawal by plasmapheresis. The IVIG role in the treatment of AMR is more complex than just diluting antibodies, as proposed in several studies (1). The IVIG exerts immunomodulatory effects on B- and T-cells at high dosage. The IVIG may induce B-cell apoptosis and modulates B-cell signaling (22). It also inhibits antibody binding to the allograft and complement activities, by unknown mechanisms (23). Therefore, removing high amounts of IVIG by plasmapheresis, without its replacement, due to not adding removed IVIG to the cumulative dose, may explain our high rate of treatment failure.

Due to a series of similarities in clinical and laboratory features of AMR and TMA in kidney transplant recipients, the unavailability of DSA assessment in Iranian laboratories, high risk kidney biopsy in several patients, especially in those with thrombocytopenia, and delayed pathology reports of renal biopsy in several Iranian kidney transplant centers, it seems reasonable to start AMR and TMA management concomitantly, in clinical situations. It means that it is better to start plasmapheresis with IVIG and rituximab. Due to the lack of Iranian insurance coverage of rituximab by nephrologist's order and high cost of this drug, most patients are not able to afford its cost. Based on these clinical limitations, we propose the approach to benefit maximally the available treatments. Our suggestion is to reduce IVIG dose after each plasmapheresis to $100 \mathrm{mg} / \mathrm{kg}$ (i.e. replacement dose) to reach the cumulative dose of $2 \mathrm{~g} / \mathrm{Kg}$. If plasmapheresis treatment is held sooner than the completion of IVIG cumulative dose of $2 \mathrm{~g} / \mathrm{kg}$, the remaining dose can be administered during one injection.

\section{Acknowledgements}

This study is a part of a Pharmacy Doctorate Thesis supported by Tehran University of Medical Sciences, Tehran, Iran. Authors would like to appreciate the help of all nurses at the Kidney Transplant Ward of Imam-Khomeini Hospital Complex, Tehran, Iran.

\section{References}

1. Levine MH, Abt PL. Treatment options and strategies for antibody mediated rejection after renal transplantation. Semin Immunol. 2012;24(2):136-42.

2. Lefaucheur C, Loupy A, Vernerey D, Duong-Van-Huyen JP, Suberbielle C, Anglicheau D, et al. Antibody-mediated vascular rejection of kidney allografts: a population-based study. Lancet. 2013;381(9863):313-9.
3. Barba J, Zudaire JJ, Robles JE, Rosell D, Berian JM, Pascual I. Complications of kidney transplantation with grafts from expanded criteria donors. World J Urol. 2013;31(4):893-900.

4. Amer H, Sibulesky L, Wadei HM. Complications of Kidney Transplantation.New York: Springer; 2013.

5. White NB, Greenstein SM, Cantafio AW, Schechner R, Glicklich D, McDonough P, et al. Successful rescue therapy with plasmapheresis and intravenous immunoglobulin for acute humoral renal transplant rejection. Transplantation. 2004;78(5):772-4.

6. Djamali A, Kaufman DB, Ellis TM, Zhong W, Matas A, Samaniego M. Diagnosis and management of antibody-mediated rejection: current status and novel approaches. Am J Transplant. 2014;14(2):255-71.

7. Schinstock C, Stegall MD. Acute Antibody-Mediated Rejection in Renal Transplantation: Current Clinical Management. Curr Transplant Rep. 2014;1(2):78-85.

8. Colvin RB. Antibody-mediated renal allograft rejection: diagnosis and pathogenesis. J Am Soc Nephrol. 2007;18(4):1046-56.

9. John R, Herzenberg AM. Our approach to a renal transplant biopsy. J Clin Pathol.2010;63(1):26-37.

10. Gloor JM, Winters JL, Cornell LD, Fix LA, DeGoey SR, Knauer RM, et al. Baseline donor-specific antibody levels and outcomes in positive crossmatch kidney transplantation. Am J Transplant. 2010;10(3):582-9.

11. Kim M, Martin ST, Townsend KR, Gabardi S. Antibody-mediated rejection in kidney transplantation: a review of pathophysiology, diagnosis, and treatment options. Pharmacotherapy. 2014;34(7):733-44.

12. Montgomery RA, Cozzi E, West LJ, Warren DS. Humoral immunity and antibody-mediated rejection in solid organ transplantation. Semin Immunol. 2011;23(4):224-34.

13. Roberts DM, Jiang SH, Chadban SJ. The treatment of acute antibody-mediated rejection in kidney transplant recipients-a systematic review. Transplantation. 2012;94(8):775-83.

14. Westwood JP, Webster H, McGuckin S, McDonald V, Machin SJ, Scully M. Rituximab for thrombotic thrombocytopenic purpura: benefit of early administration during acute episodes and use of prophylaxis to prevent relapse. J Thromb Haemost. 2013;11(3):481-90.

15. Ireland R. Thrombotic microangiopathy: rituximab in severe autoimmune TTP. Nat Rev Nephrol. 2012;8(3):131.

16. Clark WF. Thrombotic microangiopathy: current knowledge and outcomes with plasma exchange. Semin Dial. 2012;25(2):214-9.

17. George JN, Nester CM. Syndromes of thrombotic microangiopathy. N Engl J Med. 2014;371(7):654-66.

18. Froissart A, Buffet M, Veyradier A, Poullin P, Provot F, Malot S, et al. Efficacy and safety of first-line rituximab in severe, acquired thrombotic thrombocytopenic purpura with a suboptimal response to plasma exchange. Experience of the French Thrombotic Microangiopathies Reference Center. Crit Care Med. 2012;40(1):104-11.

19. Kremer Hovinga JA, Vesely SK, Terrell DR, Lammle B, George JN. Survival and relapse in patients with thrombotic thrombocytopenic purpura. Blood. 2010;115(8):1500-11.

20. Noris M, Remuzzi G. Thrombotic microangiopathy after kidney transplantation. Am J Transplant. 2010;10(7):1517-23.

21. Jodele S, Laskin BL, Goebel J, Khoury JC, Pinkard SL, Carey PM, et al. Does early initiation of therapeutic plasma exchange improve outcome in pediatric stem cell transplant-associated thrombotic microangiopathy? Transfusion. 2013;53(3):661-7.

22. Kazatchkine MD, Kaveri SV. Immunomodulation of autoimmune and inflammatory diseases with intravenous immune globulin. N Engl J Med. 2001;345(10):747-55.

23. Frank MM, Miletic VD, Jiang H. Immunoglobulin in the Control of Complement Action. Immunol Res. 2000;22(2-3):137-46. 